\title{
Risk Analysis for Dangerous Chemicals Highway Transportation Based On Adjoint Method
}

\author{
Jing $\mathrm{LI}^{1}$, Long ZHOU ${ }^{1}$, Feng LIU, Shun-Xiang HUANG*and Zhu-Lei ZHOU \\ The Institute of NBC Defense, Beijing 102205, China \\ ${ }^{1}$ These authors contributed equally to this work and both should be given the status of first author \\ * Corresponding author. \\ Tel.: 86-1066756362 \\ E-mail address: shunxianghuang@163.com
}

Keywords: Highway transportation; risk analysis; adjoint method; dangerous chemicals

\begin{abstract}
It is key to assess quantitatively the transportation risks of hazardous materials for optimal route. It's difficult to analyze efficiently transportation risk involved the factors such as meteorological conditions, the underlying properties and sensitivity to harm. So the adjoint method is used to analyze transportation risks, the adjoint equation to calculate poisoning dose is deduced based on advection diffusion equation, the transportation risks, individual risks and social risks integrated with traditional risk model and principles of probability statistics were respectively analyzed, and penalty function is used to handle the constraint condition. The case suggested the feasibility of this method. In this paper, the dynamic model is proposed, which reflected the correlation between these factors such as meteorological conditions, topography conditions, toxicity of hazardous materials and transportation risks. The adjoint method is developed to use to solve this problem. These basic theories are meaningful for risk control of dangerous chemicals transportation.
\end{abstract}

\section{Introduction}

The transport risks of dangerous chemicals with the characteristics of "highly toxic, explosive and flammable" is a major threat which could bring tremandous hazards to the residents, the economy and the environment along the transport path once an accident occurs. Accidents of dangerous chemicals transportation such as the liquefied gas leak in Hangyong highway in 2004, the liquid chlorine leak on Beijing-Shanghai highway in 2005, and the liquid nitrogen leak in 2011, in Songyang of Zhejiang province, which all attracted wide public attentions. Therefore, doing a good job in the control of transportation for dangerous chemicals is an important social security issues, and the risk analysis of transportation for dangerous chemicals is an important basis and key support for the decision-making of transportation safety, accident warning and emergency rescue. In recent years, scholars from domestic and abroad have carried out a lot of researches about risk analysis of transportation for dangerous chemicals and proposed a variety of risk measurement models in classical risks, perceived risks and condition risks considering the different degrees of risk aversion[1]. The risk characterization methods are all basically improved based on the classical risk model[2]. But the differences of accident consequence assessment are bigger. Most research results are obtained by the gauss model or regarding the hazard areas as some kind of shapes to evaluate accident consequences by chemical leakage. And in most studies they have only considered the harm to the population, not the impact on the accident consequences by the ecological environment, economic conditions, emergency levels, meteorological factors and the properties of the underlying surface of transport areas, obviously can't objective characterization of transportation risk, which obviously cannot objectively represent the transportation risks. Although the problems above can be solved by using the computers to solve the advection diffusion equations for many times, the calculation workload is too much so that the timeliness would be compromised. The successful application of the adjoint method in industrial layout, air quality control, engineering of chemical weapons destruction and other fields[3] has provided a reference for solving this problem. In this paper, the adjoint method is 
used for risk assessment on transportation of dangerous chemicals to build the adjoint model on the basis of CAMx air pollution model, which is used for the quantification of poison dose by chemical leak. Taking the differences of hazard sensitivity in the transportation area into consideration for risk measurement can effectively solve the problem caused by insufficient computing capacity, lack of timeliness and the misalignment of assessment results

\section{The Building of the Adjoint Model Based On Camx}

The adjoint method can be used to rapidly and quantitatively evaluate the risks of non-fixed hazardous chemicals, and the key is to construct appropriate adjoint equations. The extent of hazard by dangerous chemical leakage depends on the material type, weather, terrain and the location of the leakage. First, the quantitative expression of toxic dose in Eq. 1 is established, which is expressed as the integral of the space and time of the assessment areas. The integrand is the product of the hazard concentration and the weight factor, which is

$$
J=\int_{t_{0}}^{t_{0}+T} \mathrm{~d} t \iiint_{\Omega} p(x, y, z) \mathrm{C}(x, y, z, t) d \Omega
$$

Where $J$ is the toxic dose. $P$ is the weight coefficient which represents the hazard sensitivity of protected areas and has connections with the population density, environmental sensitivity, economic conditions, emergency response ability and other related factors in a certain area. It is a relative concept and usually obtained by analytic hierarchy process (ahp)[4]. $C$ is the hazard concentration which is a function of time and space. $t_{0}$ is the time of the accident. $T$ is the exposure time reflecting the level of the emergency. $\Omega$ is the domain of integration. The equation operator is introduced here

$$
L=u \frac{\partial}{\partial x}+v \frac{\partial}{\partial y}+\omega \frac{\partial}{\partial z}-K_{H}\left(\frac{\partial^{2}}{\partial x^{2}}+\frac{\partial^{2}}{\partial y^{2}}\right)-\frac{1}{D^{2}} \frac{\partial}{\partial z} K_{v} \frac{\partial}{\partial z}
$$

The equation of advection diffusion can be represented as

$$
\frac{\partial C}{\partial t}+L C=S
$$

The adjoint operator has already been derived in the literature of Liu feng et al.[5]

$$
L^{*}=-u \frac{\partial}{\partial x}-v \frac{\partial}{\partial y}-\omega \frac{\partial}{\partial z}-\left(\frac{\partial u}{\partial x}+\frac{\partial v}{\partial y}+\frac{\partial \omega}{\partial \bar{z}}\right)-K_{H}\left(\frac{\partial^{2}}{\partial x^{2}}+\frac{\partial^{2}}{\partial y^{2}}\right)-\frac{1}{D^{2}} \frac{\partial}{\partial \bar{z}} K_{v} \frac{\partial}{\partial \bar{z}}
$$

The adjoint variable $C^{*}$ is introduced to define the adjoint equation

$$
-\frac{\partial C^{*}}{\partial t}+L^{*} C^{*}=P
$$

Now we substitute Eq. 5 into Eq. 1

$$
\begin{gathered}
J=\int_{t_{0}}^{t_{0}+T} \mathrm{~d} t \iiint_{\Omega} \mathrm{C}\left(-\frac{\partial C^{*}}{\partial t}+L^{*} C^{*}\right) d \Omega \\
=\int_{t_{0}}^{t_{0}+T}\left[\iiint_{\Omega} \mathrm{C} \square L^{*} C^{*} d \Omega\right] \mathrm{d} t+\iiint_{\Omega}\left[-\int_{t_{0}}^{t_{0}+T} \frac{\partial C^{*}}{\partial t} \square C \mathrm{~d} t\right] d \Omega
\end{gathered}
$$

According to the properties of the adjoint operator, the first term on the right of Eq. 6 is derived as

$$
\int_{t_{0}}^{t_{0}+T}\left[\iiint_{\Omega} C \square L^{*} C^{*} d \Omega\right] \mathrm{d} t=\int_{t_{0}}^{t_{0}+T}\left[\iiint_{\Omega} C^{*} \square L C d \Omega\right] \mathrm{d} t
$$

The second term on the right is derived as 


$$
\iiint_{\Omega}\left[-\int_{t_{0}}^{t_{0}+T} \frac{\partial C^{*}}{\partial t} \llbracket C \mathrm{~d} t\right] d \Omega=\iiint_{\Omega}\left[-\left.C C^{*}\right|_{t=t_{0}} ^{t=t_{0}+T}+\int_{t_{0}}^{t_{0}+T} \frac{\partial C}{\partial t} \sqsubset C^{*} \mathrm{~d} t\right] d \Omega
$$

Considering Eq. 3 and the the definite conditions we finally get

$$
J=\int_{t_{0}}^{t_{0}+T} \mathrm{~d}_{t} \iiint_{\Omega} S(r, t) C^{*}\left(r, t_{0}\right) d \Omega+\iiint_{\Omega} \mathrm{C}_{0}(r) C^{*}\left(r, t_{0}\right) d \Omega
$$

As we can see from Eq. 9, the risk function can be expressed as a function of the source $S$, the initial concentration $C_{0}$ and the adjoint variable $C^{*}$. The time and space distribution of the adjoint variable $C^{*}$ meets Eq. 5, and the solution of the adjoint equation has no relationship with the location and concentration distribution of the source. Therefore, if the distribution of the associated variables $C^{*}$ is known, we can use Eq. 9 to build the adjoint model based on the CAMx air pollution model, which is easy to calculate the poisoning dose of the position.

As shown in fig. 1, the hazard sensitivity of the protected area within a certain transport region is given (namely the value distribution of $P$ ), and as shown in fig. 2, the poisoning dose distribution of the protected area by the transport of a certain amount of dangerous chemicals is abtained throught the caculatin by the adjoint model.

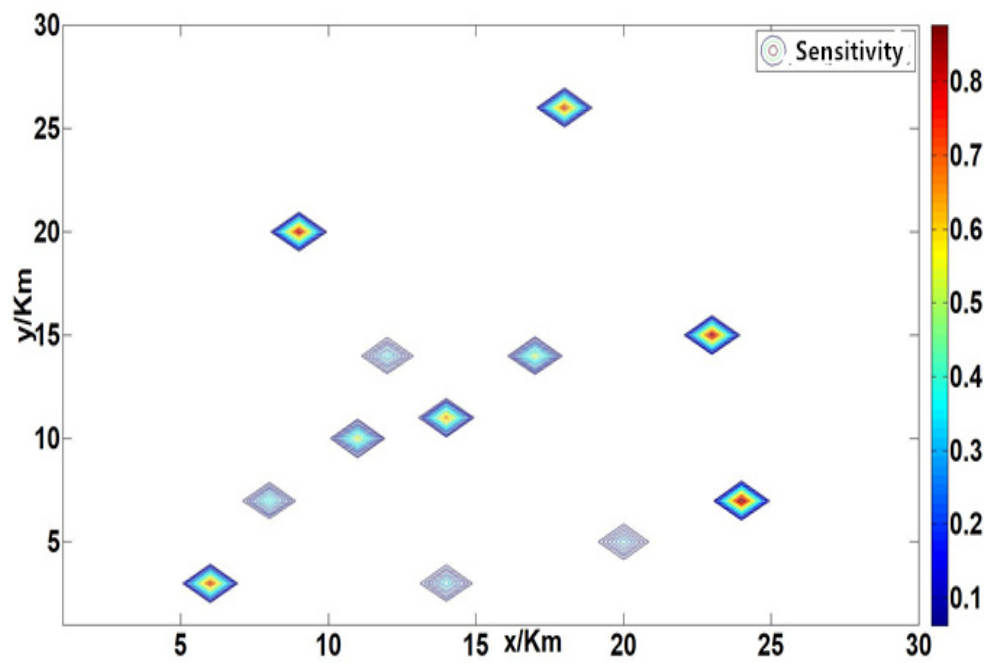

Fig. 1 The distribution of hazard sensitivity of the protected area (Relative value)

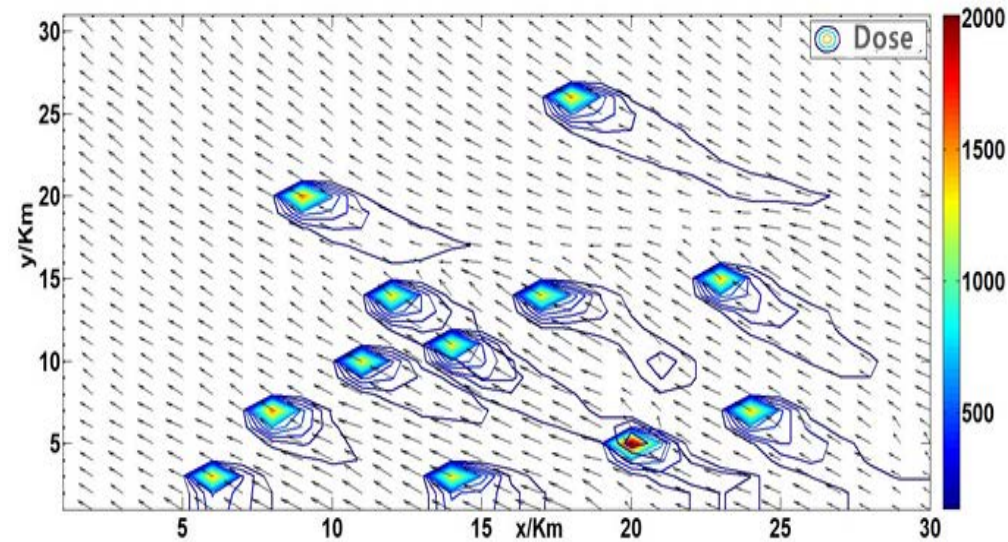

Fig. 2 The poisoning dose distribution of the protected area (unit: $\mathrm{mg} . \mathrm{s} / \mathrm{m}^{3}$ )

\section{Calculation of Transportation Risks of Dangerous Chemicals}

\section{Characterization Equation of Transportation Risks}

Because of the non-linear positive correlation between the poisoning dose and the accident consequence, the accumulation of the poisoning dose in the same area will inevitably lead to the 
aggravation of the accident consequence. Based on the Principle of probability statistics, the poisoning dose is converted by a equation of mild injury rate, and the relationship euqation[6] of mild injury rate $D_{t}$ and toxic dose is.

$$
D_{t}(x)=1 /\left[1+(x+0.81)^{-(\alpha-\beta x)}\right]
$$

Where $D_{t}$ is the minor injury rate of the protected area. $x$ represents the number of medial lethal dose, which is the ratio of the maximum toxic dose $L_{c t}^{\prime}$ in the protected area to the level of medial lethal dose $L_{c t 50}$. The toxic dose level $L_{c t 50}, \alpha, \beta$ of medial lethal is related to the dangerous chemicals themselves. The maximum toxic dose is calculated by the adjoint model and gaussian distribution equations.

For the unit accident rate $f_{(i, j)}$ of the transportation route within any grid $(i, j)$, the accident consequence is $D_{(i, j)}^{\prime}$. According to the classical risk model[2], the transportation risk at some point on the transportation route is.

$$
P_{(i, j)}=f_{(i, j)} D_{(i, j)}^{\prime}
$$

The transportation risk of the $\operatorname{grid}(i, j)$ is

$$
R_{(i, j)}=\int f_{(i, j)} D_{(i, j)}^{\prime} d l_{(i, j)}
$$

The transportation risk of hazardous chemicals is represented as

$$
R=1-\prod\left(1-\int f_{(i, j)} D_{(i, j)}^{\prime} d l_{(i, j)}\right)
$$

If we assume that risk of a point $P_{(i, j)}(l)$ in the grid $(i, j)$ is continuous on the route $l$. For the convenience of expression, it is represented as the limits of both sides of transport risk Eq. 13

$$
\lim _{\Delta l \rightarrow 0}(R)=\lim _{\Delta l \rightarrow 0}\left(1-\prod\left(1-\int f_{k} D_{k}^{\prime} d l_{k}\right)\right)
$$

So:

$$
1-\lim _{\square l \rightarrow 0}(R)=\exp \left\{\sum_{k=1}^{N} \ln \left[\left(1-\int f_{k} D_{k}^{\prime} d l_{k}\right)\right]\right\}=\exp \left\{\sum_{k=1}^{N} \ln \left[\left(1-R_{k}\right)\right]\right\}
$$

We perform equation transformation according to Taylor's formula

$$
\sum_{k=1}^{N} \ln \left[\left(1-R_{k}\right)\right]=-\sum_{k=1}^{N}\left(R_{k}+\frac{1}{2} R_{k}^{2}+\frac{1}{3} R_{k}^{3}+\cdots\right)
$$

Where

$$
\begin{aligned}
\sum_{k=1}^{N}\left(\frac{1}{2} R_{k}^{2}+\frac{1}{3} R_{k}^{3}+\cdots\right) \leq & \frac{1}{2} \sum_{k=1}^{N}\left(R_{k}^{2}+R_{k}^{3}+R_{k}^{4}+\cdots\right) \\
& \leq \frac{N}{2}\left(R_{\max }^{2}+R_{\max }^{3}+R_{\max }^{4}+\cdots\right)=\frac{N}{2} \frac{R_{\max }^{2}}{1-R_{\max }}
\end{aligned}
$$

We take the limits on both sides of Eq. 16

$$
\lim _{\Delta l \rightarrow 0} \sum_{k=1}^{N} \ln \left[\left(1-R_{k}\right)\right]=-\lim _{\Delta l \rightarrow 0} \sum_{k=1}^{N}\left(R_{k}\right)-\lim _{\Delta l \rightarrow 0} \sum_{k=1}^{N}\left(R_{k}\right)-\lim _{\Delta l \rightarrow 0} \sum_{k=1}^{N}\left(\frac{1}{2} R_{k}^{2}+\frac{1}{3} R_{k}^{3}+\cdots\right)
$$

Where the first term of Eq. 18 is transformed into 


$$
-\lim _{\Delta l \rightarrow 0} \sum_{k=1}^{N}\left(R_{k}\right)=-\int_{l} P(l) d l
$$

And the second term of Eq. 18is transformed into

$$
-\frac{N}{2} \frac{R_{\max }^{2}}{1-R_{\max }} \leq-\lim _{\Delta l \rightarrow 0} \sum_{k=1}^{N}\left(R_{k}\right)-\lim _{\Delta l \rightarrow 0} \sum_{k=1}^{N}\left(\frac{1}{2} R_{k}^{2}+\frac{1}{3} R_{k}^{3}+\cdots\right) \leq 0
$$

Because

$$
\lim _{\Delta l \rightarrow 0}\left(-\frac{N}{2} \frac{R_{\max }^{2}}{1-R_{\max }}\right)=\lim _{\Delta l \rightarrow 0}\left(-\frac{N}{2} \frac{p_{\max }^{2} \Delta l^{2}}{1-p_{\max } \Delta l}=0\right.
$$

By the Squeeze Theorem, the limit of the second term is 0. Therefore

$$
\lim _{\Delta l \rightarrow 0} \sum_{k=1}^{N} \ln \left[\left(1-R_{k}\right)\right]=-\int_{l} P(l) d l
$$

So

$$
R=1-\exp \left(-\int_{l} P(l) d l\right)
$$

\section{Characterization of Personal Risks and Social Risks}

Personal risk refers to the probability that a person who has not taken any protective measures suffers a particular harm at a particular location within a certain period of time, and the harm usually means death. Social risk refers to the probability or frequency of the expected occurrence of an accident or a group of accidents that are not below a certain severity within a certain period of time. According to the meaning of personal risk and social risk, the personal risk and social risk model of dangerous chemical transportation in the adjoint model are characterized as follow.

Hazardous chemicals are transported on the transportation route s. For the protected areas, the transportation sections that may pose threats to the area c are ab, and the personal risk of the area c is

$$
I R_{c}=\int_{a b}\left(f_{S} D_{l S}\right) d s
$$

Where $f_{S}$ is the transportation accident rate per unit length of the section. $D_{I S}$ is the casualty rate of the area caused by unit road section, and the casualty rate equation[6] is

$$
D_{l}(x)=1 /\left[1+x^{-(\gamma+\theta x)}\right]
$$

And $x, \gamma, \theta$ are related to the properties of dangerous chemicals themselves.

We assume that the values of $f_{\mathrm{s}}$ in the same grid are equal, the casualty rate of the grid $(i, j)$ on the region c can be represented as

$$
I R_{c_{(i, j)}}=\int\left(f_{(i, j)} D_{l(i, j)}\right) d_{s}=f_{(i, j)} \int\left(D_{l(i, j)}\right) d s
$$

Since the casualty rate $D_{l(i, j)}$ of all points in the same grid to the region is equal, Eq. 26 can be transformed into

$$
I R_{c_{(i, j)}}=f_{(i, j)} D_{l(i, j)} l_{(i, j)}
$$

Where $l_{(i, j)}$ is the length of the route $\mathrm{s}$ in the grid $(i, j)$, and the value of the toxic dose $L_{c t}$ in the region c is determined by the adjoint model. 
Because the individual risks are equal at any location in the region c, the social risks $S R_{\mathrm{c}}$ are represented as follow according to the meaning of social risks.

$$
S R_{\mathrm{c}}=I R_{\mathrm{c}} \cdot P O P_{\mathrm{c}}
$$

Where $P O P_{\mathrm{c}}$ is the total population of the region $\mathrm{c}$.

\section{Characterization of Equivalent Transportation Risks}

The transportation management department clearly stipulates when transporting dangerous chemicals, the transportation risk of a certain section of the road generally needs to meet the constraints of standards of the personal risk and social risk. If there is no alternative route, safety control must be strengthened. In the calculation of transportation risk, the penalty function can be used to deal with these constraint conditions. The value of penalty function is small when the constraints are satisfied and significantly increases when the constraints are not satisfied. The following penalty function is defined focusing on the personal risk and social risk

$$
M_{1} \max \left(\left(1-I R_{S t} / I R_{C}\right), 0\right) \quad M_{2} \max \left(\left(1-S R_{S t} / S R_{C}\right), 0\right)
$$

In which $M_{1}$ and $M_{2}$ are the penalty factor, and in terms of the strict constraints $M_{1}$ and $M_{2}$ should be towards infinity. However, for the needs of computer operation and actual transportation, large values or values with monotonic changes are usually taken. In order to show the monotone increasing relation of equivalent risk and the degree of threats, we define

$$
M(x)=\kappa x^{2} /(1-\lambda x)
$$

In this equation, $x=1-I R_{S t} / I R_{C}$ or $x=1-S R_{S t} / S R_{C}, \kappa$ is a number greater than 1 , and the greater the value is the more obvious for the penalty of the function. $\lambda$ is a positive number not greater than 1 , and the greater the value is the more obvious for the penalty of the function. The equivalent risk of transiportaion can be defined as

$$
F^{\prime}=\sum_{(i, j) \in E} X_{i j} R^{(i, j)} \square\left(1+M_{1} \max \left(\left(1-I R_{s t} / I R_{c}\right), 0\right)\right) \llbracket\left(1+M_{2} \max \left(\left(1-S R_{s t} / S R_{c}\right), 0\right)\right)
$$

\section{An Example}

Suppose there is a need to transport 456.8kg of Japanese acws (abandoned chemical weapons) to other places for destruction. The transportation network between the two places is shown in figure 3 , and there are 12 protected areas between the two places (star shape).Assume that the transportation road traffic accident rate, path length, the traffic speed and the hazard sensitivity within the set grid are all known, as shown in figure 1 . Then the problem is how to choose a path from the network with minimum risks.

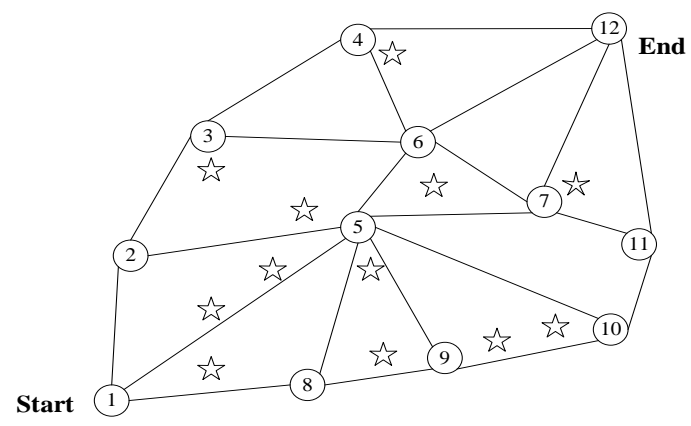

Fig. 3 Transportation network in transportation area

The adjoint model constructed based on the adjoint Eq. 9 can obtain the toxic dose distribution in the protected area, which is shown in figure 1. In the Matlab, the transportation risks of different 
sections to all protected areas are obtained by Eq. 10 and Eq. 23, as shown in table 1. And by using Eq. 24, 26 and 27, we can obtain the personal risks of different sections in all protected areas, as shown in table 2. On the basis of personal risks, the social risks of different sections by transportation to all protected areas can be obtained by using Eq. 28.

Table 1 transportation risks in all protected areas on different roads

\begin{tabular}{cccccc}
\hline $\begin{array}{c}\text { Section } \\
\text { Name }\end{array}$ & $\begin{array}{c}\text { Transport risk } \\
\left(\times 10^{-5}\right)\end{array}$ & $\begin{array}{c}\text { Section } \\
\text { Name }\end{array}$ & $\begin{array}{c}\text { Transport risk } \\
\left(\times 10^{-5}\right)\end{array}$ & $\begin{array}{c}\text { Section } \\
\text { Name }\end{array}$ & $\begin{array}{c}\text { Transport risk } \\
\left(\times 10^{-5}\right)\end{array}$ \\
\hline R01-02 & 1.30 & R04-12 & 1.70 & R07-11 & 0.69 \\
R01-05 & 3.67 & R05-06 & 0.61 & R07-12 & 2.91 \\
R01-08 & 1.16 & R05-07 & 2.79 & R08-09 & 0.57 \\
R02-03 & 4.23 & R05-08 & 2.62 & R09-10 & 1.00 \\
R02-05 & 4.27 & R05-09 & 0.95 & R10-11 & 0.53 \\
R03-04 & 1.68 & R05-10 & 4.12 & R11-12 & 1.96 \\
R03-06 & 2.78 & R06-07 & 0.86 & & \\
R04-06 & 0.81 & R06-12 & 1.45 & & \\
\hline
\end{tabular}

Since the personal risks of R01-05 sections exceeds the acceptable risk $\left(5 \times 10^{-6}\right)$, the punishment coefficient is 43.8 caculated by Eq. 30 when taking $\kappa=100$ and $\lambda=0.5$. Then the equivalent transportation risk of this section is obtained by using Eq. 31, which is $1.64 \times 10^{-3}$ and the transportation risks of the remaining sections is lower than the acceptable standard, and the equivalent transportation risks are equal to the transportation risks. Through this the distribution of equivalent transport risk matrix among nodes of transport routes can be obtained. It is not difficult to conclude that the route with the lowest transportation risk starts from the starting point and reaches the end point after nodes 8, 9, 10 and 11.

Table 2 personal risks to all protected areas on different road

\begin{tabular}{cccccc}
\hline $\begin{array}{c}\text { Section } \\
\text { Name }\end{array}$ & $\begin{array}{c}\text { Personal } \\
\text { risk }\left(\times 10^{-6}\right)\end{array}$ & $\begin{array}{c}\text { Section } \\
\text { Name }\end{array}$ & $\begin{array}{c}\text { Personal } \\
\operatorname{risk}\left(\times 10^{-6}\right)\end{array}$ & $\begin{array}{c}\text { Section } \\
\text { Name }\end{array}$ & $\begin{array}{c}\text { Personal } \\
\text { risk }\left(\times 10^{-6}\right)\end{array}$ \\
\hline R01-02 & 4.25 & R04-12 & 0.30 & R07-11 & 0.07 \\
R01-05 & 11.40 & R05-06 & 0.08 & R07-12 & 1.06 \\
R01-08 & 0.04 & R05-07 & 0.64 & R08-09 & 0.03 \\
R02-03 & 2.12 & R05-08 & 1.01 & R09-10 & 0.06 \\
R02-05 & 1.29 & R05-09 & 0.06 & R10-11 & 0.01 \\
R03-04 & 0.91 & R05-10 & 3.28 & R11-12 & 0.19 \\
R03-06 & 1.14 & R06-07 & 0.05 & & \\
R04-06 & 0.05 & R06-12 & 0.27 & & \\
\hline
\end{tabular}

\section{Conclusion}

The adjoint model based on CAMx air pollution model can be applied to transport risk analysis, which can significantly improve the efficiency and precision of risk analysis and expand the application field of adjoint method. The quantification of transportation risks is given based on the characterization method of classical risk model, and a fast solution method is developed by strict mathematical derivation. We calculate the equivalent transportation risk by introducing penalty function, the personal and social risk standard constraints are considered. Finally, an example is given to verify the effectiveness of this method. Based on the internal connection of the meteorological conditions, terrain conditions, the toxicity of dangerous materials and other factors with the transportation risk, we built the dynamic model and prospose a fast algorithm solution, which provides a basic theory for the risk prevention and control of dangerous chemicals transportation. 


\section{Acknowledgement}

This study is supported by National Key Research and Development Program of China under grant 2016YFC0209000 and National Natural Science Foundation of China under grants 41375154. The wind field simulations were conducted using the National Air quality high Resolution forecasting and pollution control decision Support system(NARS).

\section{References}

[1] ZHANG J H, ZHU D L. Review on risk analysis of dangerous chemical transportation [J]. China Safety Science Journal, 2007, 27(3):136-140

[2] A. Ang, J. Briscoe. Developing of a systems risk methodology for single and multimodal transportation systems[R]. Ishington,D.C. Office of University Research, U.S. Department of Transportation,1989

[3] Liu Feng, Hu Fei, Zhu Jiang, et al. Some new progresses in the theory for optimal control of atmospheric pollution [J]. Study and development on technology, 2004, 26(6): 7-14

[4] Jing Li, Xuezhi Zhou, Shunxiang Huang,et al. Transportation risk analysis for chemical agents based on adjoint method. 2011 International Conference on Electrical and Control Engineering. 2011: 5625-5628

[5] LIU F, HU F. Inversion of diffusion coefficients and effect of related difference schemes [J]. Journal of Applied Meteorological Science, 2003, 14(3): 331-338

[6] HUANG S X, CHEN H P, LIU F. Chemical risk assessment [M]. Beijing: China Meteorological Press, 2009 